\title{
MINERAL IDENTIFICATION IN LWIR HYPERSPECTRAL IMAGERY APPLYING SPARSE SPECTRAL CLUSTERING
}

\author{
Bardia Yousefi $^{1 *}$, Clemente Ibarra Castanedo ${ }^{1}$, Georges Beaudoin ${ }^{2 *}$, Xavier P V Maldague $^{1+*}$, François Huot $^{2}$, \\ Martin Chamberland ${ }^{3}$
}

\author{
${ }^{1}$ Computer vision and systems laboratory, Department of Electrical and Computer Engineering, Laval \\ University, 1065, av. de la Médecine, Quebec City (Quebec) G1V 0A6, Canada. bardia.yousefi.1@ulaval.ca \\ ,\{IbarraC, Xavier.Maldague\}@gel.ulaval.ca. \\ ${ }^{2}$ Department of Geology and Geological Engineering, Laval University, 1065, av. de la Médecine, Quebec City \\ (Quebec) G1V 0A6, Canada. \{ Georges.Beaudoin,Francois.Huot \} @ ggl.ulaval.ca. \\ ${ }^{3}$ Telops, Inc., 100-2600 St-Jean-Baptiste Ave, Quebec City, Qc, G2E 6J5, Canada. \\ martin.chamberland@telops.com
}

${ }^{*}$ Corresponding Author, ${ }^{+*}$ Presenting and Corresponding Author

\begin{abstract}
The application of hyperspectral infrared imagery has considerably increased in the different fields of research. Hyperspectral image analysis is mainly used in remote sensing for various applications such as target detection, vegetation detection, urban area categorization, astronomy and geology. The mineralogical applications of this technology involves mineral identification in remote sensing, airborne imagery, portable instruments, and core logging. Recently, hyperspectral imagery has been used in laboratory conditions for mineral grains identification.

We address a complete assessment (quantitative and qualitative) of mineral identification in the laboratory conditions and try to identify nine different minerals (biotite, diopside, epidote, goethite, kyanite, scheelite, smithsonite, tourmaline, quartz). A hyperspectral camera in the Long-Wave Infrared (LWIR, 7.7-11.8 $\mu \mathrm{m}$ ) with a LW-macro lens, an infragold plate, and a heating source are the instruments used in the experiment. For identification, a Sparse Principal Component Analysis (SPCA) based Kmeans clustering (Sparse spectral clustering) is employed to group all the pixel-spectra in different groups. Then the best representatives of each cluster are chosen to compare with the ASTER spectral library of JPL/NASA through spectral comparison techniques. Spectral angle mapper (SAM) and Normalized Cross Correlation (NCC) are two of such techniques, which are used herein to measure the spectral difference. In order to evaluate the robustness of the clustering results among the mineral's spectra, we have added three levels of Gaussian noise, $0 \%, 2 \%$, and $4 \%$, to input spectra which dropped the accuracy percentage $34.17 \%$ from $73.54 \%$, for $0 \%$ to $2 \%$ additive noise, and $19.1 \%$, for $2 \%$ to $4 \%$ additive noise. The results conclusively indicate the promising performance but noise sensitive behavior of the proposed approach.
\end{abstract}

KEYWORDS: hyperspectral imagery, mineral identification, sparse principle components analysis, spectral clustering.

\section{SUMMARY}

Clustering approaches have been proposed for countless applications in different research areas such as pattern recognition and data-mining. One of the main interest for such approaches is the inherent benefit of being an unsupervised learning method. K-Means clustering [1] methods optimize the squared error function and has been used for many categorization applications. This method was enhanced to the kernelled clustering which transforms high dimensional data to lower dimensional data to improve classification. One kernel method that is frequently used in clustering approaches is Principal Component Analysis (PCA) [2], which creates spectral clustering methods due to application of eigenvalues for dimension reduction. PCA projects the data into a lower dimensional space i.e. eigen space and picks up the dimensions that correspond to the largest variances. From the mathematical point of view, this is like approximating the best low rank of the data. PCA however, is highly affected by noise due to being a linear transformation. On the other hand, Sparse Principal Component Analysis (SPCA) [3] shows more robust behavior in front of noise as compared to PCA. Hence, SPCA based clustering should theoretically lead to a better performance. In this paper we address a quantitative and qualitative assessment of mineral identification applying sparse spectral clustering in laboratory condition experiments, in the LWIR (7.7-11.8 $\mu \mathrm{m})$ and strive to identify nine different minerals (biotite, diopside, epidote, goethite, kyanite, scheelite, smithsonite, tourmaline, quartz). 


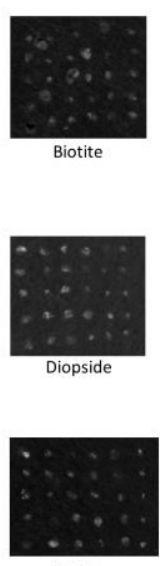

Epidote

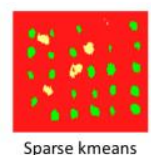

Sparse kmeans

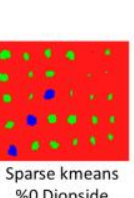

$\% 0$ Diopside

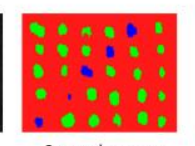

Sparse kmeans
$\% 0$ Epidote
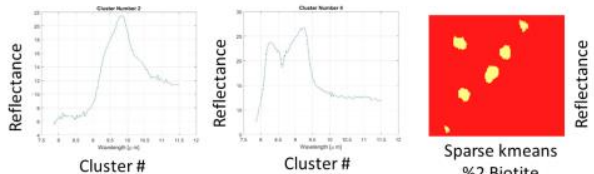

\% Biotite
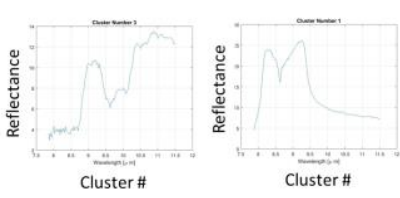

Cluster \#

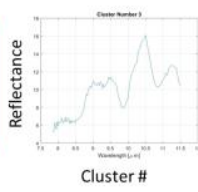

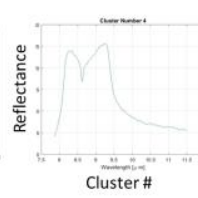
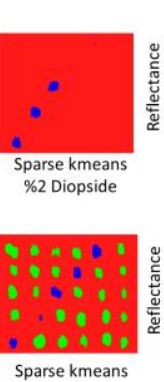

Sparse kmeans
$\% 2$ Epidote

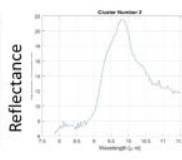

Cluster \#

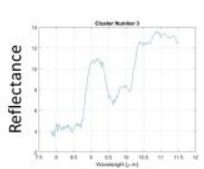

Cluster \#

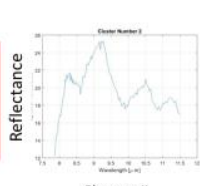

Cluster \#
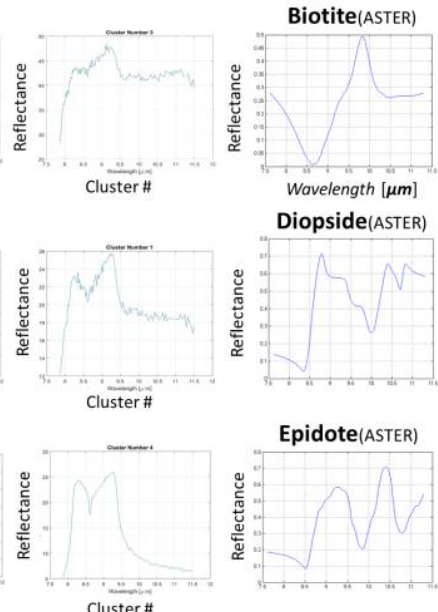

Fig.1 Mineral identification results using SAM [5] are shown for biotite, diopside, and epidote grains for $0 \%$ and $2 \%$ of additional noise along with their spectra are presented. Last columns shows the ASTER spectral library [4] for each of these minerals.

\begin{tabular}{|l|c|c|c|c|c|c|c|c|c|}
\hline \multirow{2}{*}{ Mineral } & $\begin{array}{c}\text { Spatial } \\
\text { resolution } \\
\text { of RoI }\end{array}$ & \multicolumn{2}{|c|}{ GT } & \multicolumn{4}{c|}{ Accuracy (\%) } & \multicolumn{3}{c|}{$\begin{array}{c}\text { Computaional Complexity } \\
\text { (Noise 0\%) }\end{array}$} \\
\cline { 3 - 10 } & & Mineral & Quartz & Noise (0\%) & Noise (2\%) & Noise (4\%) & $\begin{array}{c}\text { Clustering } \\
\text { time (s) }\end{array}$ & SAM (s) & NCC (s) \\
\hline Biotite & $123 * 138$ & 434 & 483 & $>90$ & 67.39 & $<10$ & 0.74815 & 0.262 & 0.299 \\
\hline Diopside & $126 * 143$ & 253 & 705 & 70.44 & 15.546 & 55.92 & 1.0188 & 0.139 & 0.235 \\
\hline Epidote & $123 * 148$ & 216 & 706 & 77.33 & 28.95 & $<10$ & 0.43088 & 0.248 & 0.242 \\
\hline Geothite & $118^{*} 141$ & 199 & 552 & 74.01 & $<10$ & $<10$ & 0.3995 & 0.0946 & 0.101 \\
\hline Kyanite & $123 * 144$ & 68 & 510 & 77.14 & 33.71 & $<10$ & 0.38055 & 0.219 & 0.256 \\
\hline Scheelite & $123 * 158$ & 134 & 810 & 92.65 & 68.07 & 66.45 & 0.49851 & 0.207 & 0.262 \\
\hline Smithsonite & $119 * 160$ & 343 & 913 & 80.39 & 54.20 & $<10$ & 0.59569 & 0.223 & 0.231 \\
\hline Tourmaline & $58 * 80$ & 122 & 9 & 79.91 & 76.43 & $<10$ & 0.11002 & 0.201 & 0.221 \\
\hline
\end{tabular}

Table 1. Accuracy of automatic mineral identification using SAM is presented for Rigid-GT with the computational cost in every case.

Fig.1 presents some results of the mineral identification using the proposed approach and shows the biotite, diopside, and epidote grains displayed in different colors for $0 \%$ and $2 \%$ additional noise along with their spectra. The approach used first clustering then discriminate them using spectral technique [6]. To test the noise robustness, we have added three levels of Gaussian noise, $0 \%, 2 \%$, and $4 \%$, to input spectra which considerably decreased the accuracy percentage of the system from average of $73.54 \%$ to 39.36 and 20.26 (Table 1), respectively. The results indicate noise sensitivity of the approach which could be due to the low spatial resolution of region of interest (ROI) which decreases the level of Signalto-Noise-Ratio (SNR). Considering that, SNR in laboratory condition is relatively higher than hyperspectral infrared remote sensing hence the noise level is not a risk for grains segmentation. In addition, the accuracy calculation was based on counting the pixels whereas in real application aside from precise measurement even one or two pixels on the grain's surface identifies the grain mineral content and additional noise only eliminates the weak signatures from the cluster's group.

\section{REFERENCES}

[1] Hartigan, J. A., \& Wong, M. A. (1979). Algorithm AS 136: A k-means clustering algorithm. Journal of the Royal Statistical Society. Series C (Applied Statistics), 28(1), 100-108.

[2] Jolliffe, I. (2002). Principal component analysis. John Wiley \& Sons, Ltd.

[3] Zou, H., Hastie, T., \& Tibshirani, R. (2006). Sparse principal component analysis. Journal of computational and graphical statistics, 15(2), 265-286.

[4] Baldridge, A. M., Hook, S. J., Grove, C. I., \& Rivera, G. (2009). The ASTER spectral library version 2.0. Remote Sensing of Environment, 113(4), 711-715.

[5] Yuhas, R. H., Goetz, A. F., \& Boardman, J. W. (1992). Discrimination among semi-arid landscape endmembers using the spectral angle mapper (SAM) algorithm.

[6] Yousefi, B., Sojasi, S., Liaigre, K., Castanedo, C. I., Beaudoin, G., Huot, F., ... \& Chamberland, M. (2017, May). Modified algorithm for mineral identification in LWIR hyperspectral imagery. In SPIE Commercial+ Scientific Sensing and Imaging (pp. 102141H-102141H). International Society for Optics and Photonics.

[7] Lewis, J. P. (1995, May). Fast normalized cross-correlation. In Vision interface (Vol. 10,No. 1, pp. 120-123). 